\title{
Clinical study of gestational trophoblastic disease in a tertiary care hospital
}

\author{
Jayashree Mulik, Archana Choudhary*
}

Department of Obstetrics and Gynecology, Government Medical College and Hospital, Nagpur, Maharashtra, India

Received: 08 May 2020

Accepted: 01 June 2020

\section{*Correspondence:}

Dr. Archana Choudhary,

E-mail: drarchanachoudhary@rediffmail.com

Copyright: () the author(s), publisher and licensee Medip Academy. This is an open-access article distributed under the terms of the Creative Commons Attribution Non-Commercial License, which permits unrestricted non-commercial use, distribution, and reproduction in any medium, provided the original work is properly cited.

\begin{abstract}
Background: Gestational trophoblastic diseases (GTDs) had been associated with significant morbidity and mortality till recently. Wide variation in incidences have been reported worldwide. The present study was planned with the objective of determination of incidence, assessment of risk factors, clinical presentation, management protocols and outcomes in GTD cases in Indian population at a tertiary care centre.

Methods: All the diagnosed cases of GTD reporting to study centre during study period of 1.5 years were included. Detailed history taking, examination and relevant investigations $(\mathrm{Hb} \%$, blood grouping, thyroid functions, serum $\mathrm{B}-$ hCG, USG and chest X-ray) were undertaken. Suction and evacuation were done for all patients as primary mode of management and samples were sent for histopathological examination. Comprehensive follow ups were done, including weekly $\mathrm{B}$-hCG until normal for 3 consecutive weeks followed by monthly determination until the levels were normal for 6 consecutive months.

Results: Total 22 cases out of 19500 deliveries were diagnosed as GTD (incidence rate-1.13/1000 deliveries). Mean age was $23.64 \pm 3.89$ years with $50 \%$ participants being primigravida. The commonest symptom after amenorrhea 22 $(100 \%)$ was bleeding per-vaginum $15(68.2 \%)$. Maximum cases were of complete hydatidiform mole histopathology $16(72.73 \%)$, USG 19 (86.4\%). Clinical characteristics were statistically comparable between patients of complete mole and partial mole. Out of 22 cases, 1 (4.5\%) was diagnosed as GTN.

Conclusions: Early diagnosis and treatment along with regular follow up is the key in GTD. There is need to establish a centralized disease specific registry in future.
\end{abstract}

Keywords: Gestational trophoblastic diseases, Gestational trophoblastic neoplasia, Hydatidiform mole, $\beta$-hCG

\section{INTRODUCTION}

Gestational trophoblastic disease (GTD) is the term used to describe heterogeneous group of interrelated lesions that arise from abnormal proliferation of placental trophoblasts with varying tendency for spontaneous remission, local invasion and metastasis. ${ }^{1}$ Benign lesions consist of hydatidiform moles, whereas malignant lesions consist of invasive mole, placental-site trophoblastic tumour (PSTT), epithelioid trophoblastic tumour (ETT) and choriocarcinoma (CC). The term "gestational trophoblastic neoplasia" (GTN) has been applied collectively to the latter four conditions; which can progress, invade, metastasize, and lead to death if left untreated. ${ }^{1}$

The wide variation in incidence that have been reported worldwide has largely been contributed by genetic, demographic, environmental and host-related factors. Low socio-economic status, protein deficiency and malnutrition have also been found to be associated. ${ }^{2}$ Meanwhile definite environmental factors have not been found to be associated to this variation. $^{2}$ In Asia, Indonesia has the highest incidence ( 1 in 77 pregnancies and 1 in 57 deliveries). ${ }^{3}$ The incidence of GTN varies in different parts/ region from 0.6-1.1 per 1000 pregnancies 
in Europe and North America to 2 per 1000 pregnancies in Japan. ${ }^{4}$ In the middle East the incidence is found to be 1 in 160 pregnancies. $^{5}$

GTDs were historically associated with significant morbidity and mortality. Gestational trophoblastic disease now is one of the completely curable reproductive health problems. Early detection with the help of ultrasound and serum $\mathrm{B}$-hCG values is very important for treatment of the disease. It is imperative that we diagnose it at early stage and in turn prevent its malignant transformation. With this goal in mind, the present study was planned with the objective of determination of incidence, assessment of risk factors, clinical presentation, management protocols and outcomes in GTD cases in an Indian population at a tertiary care centre.

\section{METHODS}

This was an observational study conducted at a tertiary care government hospital in central India from February 2018 to August 2019 i.e., one and a half year. The study population consisted of all the pregnant women registered at the study centre. Due to low incidence of gestational trophoblastic disease, all the diagnosed cases of GTD reporting to the tertiary care hospital were included. The data for total number of GTD cases, total number of pregnancies and live births in the hospital were taken from hospital registries. Following selection criteria were adopted for the present study.

\section{Inclusion criteria}

- All the cases diagnosed as GTD either histologically or sonographically which included complete mole, partial mole, invasive mole, choriocarcinoma, PSTT and ETT.

\section{Exclusion criteria}

- All other intrauterine pregnancies

- Unwillingness to give consent for the study.

Necessary approval from the institutional ethics committee of the hospital was taken before start of the study. Eligible patients were included in the study after taking a written and informed consent. Patient's comprehensive history including age, address, chief presenting complaints, gravidity, gestational age, outcome of previous pregnancies, menstrual history were noted for all participants. Then detailed general and clinical examination was done. Size of the uterus per abdomen was compared with gestational age and ascertained if it corresponded to the weeks of gestation. Per-speculum and per vaginal examination was also done. Relevant haematological and radiological investigations which included $\mathrm{Hb} \%$, blood grouping and $\mathrm{Rh}$ typing, thyroid function test, serum $\mathrm{B}-\mathrm{hCG}$, USG and chest X-ray were undertaken. Cross matching samples were drawn for blood transfusions, if and when required. Suction and evacuation were done for all patients as a primary mode of management. The samples so obtained were sent for histopathological examination. Blood transfusions were done according to requirement of the patient either preoperatively, intraoperatively or in the post-operative period. Anti-D was given to Rh-negative women. The serum $\beta$-hCG was repeated 48 hours after evacuation.

Then the patients were counselled regarding need for follow up and use of contraception during the entire period of follow up. Follow up was done with weekly $B-$ hCG until normal for 3 consecutive weeks followed by monthly determination until the levels were normal for 6 consecutive months. The normal level of $\beta-\mathrm{hCG}$ was taken to be less than $10 \mathrm{IU} / \mathrm{L}$. At each follow up visit detailed history was taken regarding irregular vaginal bleeding, pain abdomen, headache, cough, haemoptysis, and fever etc. Clinical examination, per speculum and per vaginal examination was done to look for signs of GTN. The time to achieve the first normal $\mathrm{B}-\mathrm{hCG}$ after evacuation was noted. USG as well as colour Doppler were done when clinically indicated. GTN was diagnosed during follow up either on the basis of a rise in serum BhCG levels or histopathology or with evidence of metastasis. Those diagnosed as GTN were classified as low risk or high risk using FIGO scoring system for GTN and were duly treated with chemotherapy. ${ }^{6}$

\section{Statistical analysis}

The data was entered and analysed using MS excel software and chi-square test was applied. $\mathrm{p}<0.05$ was considered as being significant. Data confidentiality was thoroughly ensured and no individual finding was shared anywhere ever.

\section{RESULTS}

There were total 19500 deliveries at the study centre during study period. A total of 22 cases out of these were diagnosed to have GTD, providing an incidence rate of 1.13/1000 deliveries. These 22 patients were considered as participants for final analysis.

Table 1: Presentation of clinical symptoms.

\begin{tabular}{|lll|}
\hline Symptoms & Cases & Percentage \\
\hline Amenorrhoea & 22 & $100 \%$ \\
\hline Per-vaginum bleeding & 15 & $68.2 \%$ \\
\hline Pain in abdomen & 7 & $31.8 \%$ \\
\hline Passage of grapes like vesicles & 2 & $9.1 \%$ \\
\hline Hyperemesis & 2 & $9.1 \%$ \\
\hline Hyperthyroidism features & 2 & $9.1 \%$ \\
\hline
\end{tabular}

Age of the relatively young participant group ranged between 18 to 35 years (mean- $23.64 \pm 3.89$ years). A total of $50 \%(11 / 22)$ participants were primigravida in the study. Six $(27 \%)$ of patients had previous history of fullterm pregnancy while $5(23 \%)$ had at least one abortion. 
Maximum number of patient $(50 \%)$ presented in the first trimester ( $\leq 12$ weeks), $45.4 \%$ presented between $13-20$ weeks and only $1(4.5 \%)$ patient presented after 20 weeks, the mean gestational age at presentation being $12.8 \pm 3.96$ weeks. Majority $(50 \%)$ of the patients had uterine size larger than the period of gestation; $27.3 \%$ had uterine size corresponding to the period of gestation and $22.7 \%$ had uterine size smaller than the period of gestation.

Table 2: Diagnostic distribution of cases.

\begin{tabular}{|c|c|c|}
\hline Typing of case & Cases & Percentage \\
\hline \multicolumn{3}{|c|}{ On histopathology report } \\
\hline Complete mole & 16 & $72.73 \%$ \\
\hline Partial mole & 5 & $22.73 \%$ \\
\hline Invasive mole & 1 & $4.54 \%$ \\
\hline \multicolumn{3}{|l|}{ On USG report } \\
\hline Complete mole & 19 & $86.4 \%$ \\
\hline Partial mole & 3 & $13.6 \%$ \\
\hline
\end{tabular}

Regarding symptoms of the participants, amenorrhea was seen in all $22(100 \%)$ patients. The commonest symptom was bleeding per-vaginum which was seen in 15 (68.2\%) of patients. The classic symptom of passage of grape like vesicles was seen in $2(9.1 \%)$ of patients only (Table 1$)$.

The commonest blood group associated with GTD was A Rh-positive (45.5\%), with AB Rh-positive (4.5\%) being the least common. Rh-negative blood groups were not encountered in this study. The mean pre-treatment $\mathrm{Hb} \%$ was $8.75 \pm 1.36 \mathrm{gm} \%$. Only $2(9.1 \%)$ patients had severe anaemia with $\mathrm{Hb} \%$ less than $6.9 \mathrm{gm} \%$. A total of 18 $(81.8 \%)$ patients required blood transfusion with mean requirement being 1.72 units of blood per patient. Out of
22 cases, 5 (22.73\%) were hyperthyroid, while majority were having normal thyroid function functions 16 $(72.73 \%)$. One was hypothyroid. All the study participants underwent chest X-ray and it was found to be normal in all of them.

Table 3: Distribution of cases by pre-evacuation BhCG level.

\begin{tabular}{|lll|}
\hline B-hCG & Cases & Percentage \\
\hline$<10,000 \mathrm{IU} / \mathrm{L}$ & 0 & $0 \%$ \\
\hline $10,000-1,00,000 \mathrm{IU} / \mathrm{L}$ & 6 & $27.3 \%$ \\
\hline$>1,00,000 \mathrm{IU} / \mathrm{L}$ & 16 & $72.7 \%$ \\
\hline
\end{tabular}

On histopathological examination, $16(72.73 \%)$ were reported to be cases of complete hydatidiform mole (CHM) and $5(22.73 \%)$ were partial hydatidiform mole (PHM). The specimen of uterus of one patient $(1.9 \%)$ who underwent hysterectomy showed hydropic villi invading myometrium suggestive of invasive mole. No cases of choriocarcinoma, PSTT or ETT were seen in the study. Of the 22 patients who got ultrasound done, 19 $(86.4 \%)$ showed picture of complete mole (Table 2 ).

On evaluation of cases with B-hCG, majority $(72.7 \%)$ had pre-evacuation $\beta$-hCG values between 1,00,000$10,00,000 \mathrm{IU} / \mathrm{L}$, with remaining $27.3 \%$ having $\mathrm{B}-\mathrm{hCG}$ values between 10,000-1,00,000 IU/L (mean preevacuation $\beta-h C G-1.99 \pm 1.60$ lakhs IU/L). The values changed post-evacuation ( 24 hours) to a mean of $0.61 \pm 0.64$ lakh IU/L. Total $20(90.9 \%)$ patients were followed till they attained first normal value of $\beta$ hCG and the mean time taken for the same was observed to be $9.1 \pm 2.21$ weeks (Table 3 ).

Table 4: Comparison of complete mole versus partial mole.

\begin{tabular}{|llll|}
\hline Variables & CHM $($ Mean \pm SD) & PHM $($ Mean \pm SD) & p value \\
\hline Maternal age (years) & $23.31 \pm 3.21$ & $24.6 \pm 6.26$ & 0.543 \\
\hline Gestational age (weeks) & $12.33 \pm 2.09$ & $15.08 \pm 7.40$ & 0.183 \\
\hline B-hcg (lakh IU/L) & $1.92 \pm 1.79$ & $1.84 \pm 0.76$ & 0.921 \\
\hline 48 hours repeat ß-hcg (lakh IU/L) & $0.54 \pm 0.53$ & $0.92 \pm 0.97$ & 0.268 \\
\hline Time for ß-hcg normalization (weeks) & $9.57 \pm 2.40$ & $7.8 \pm 1.3$ & 0.141 \\
\hline Blood transfusion (units) & $1.50 \pm 1.09$ & $2.00 \pm 1.87$ & 0.461 \\
\hline
\end{tabular}

According to prognostic scoring scale (PSS), 40.9\% patients were in low risk and $59.1 \%$ patients were in high risk group in the study. Patients in high risk were then enrolled for chemotherapy for complete recovery. Suction evacuation was the primary mode of treatment and was undertaken in $20(90.9 \%)$ of patients. One $(9.1 \%)$ patient underwent hysterectomy in view of uterine perforation during suction evacuation with moribund status. One patient, who was at 26 weeks with partial mole, underwent preterm vaginal delivery. Complications were minimal in the present study. Three (13.6\%) patients had febrile morbidity. One $(4.5 \%)$ patient had haemorrhage and one underwent hysterectomy.

Clinical characteristics were compared between patients of complete mole and partial mole. In all the studied parameters only, non-significant difference was observed between the groups. Although the pre evacuation $B-h C G$ level and gestational age at presentation was higher in CHM patients compared to PHM, the difference was not 
statistically significant. The mean time taken for $\beta-\mathrm{hCG}$ to return to normal levels after suction evacuation was $9.57 \pm 2.40$ weeks in CHM and $7.8 \pm 1.3$ weeks in PHM which was also not statistically significant, though it was higher in CHM.

With respect to thyroid status, $17.64 \%$ of $\mathrm{CHM}$ were hyperthyroid whereas $40 \%$ of PHM patients were hyperthyroid. The need for blood transfusion was more in cases of CHM $(81.25 \%)$ with average 1.5 units required per person compared to PHM where $80 \%$ required blood transfusion with an average of 2 units per person (Table $4)$.

Out of 22 cases, 1 (4.5\%) was diagnosed as GTN. She underwent hysterectomy for uterine perforation during evacuation and histopathology of uterus showed hydropic villi invading myometrium suggestive of invasive mole. She belonged to high-risk category according to FIGO score and was started with single drug (methotrexate) chemotherapy.

\section{DISCUSSION}

Because of wide geographical variations in the incidence of gestational trophoblastic disease as a result of differences in methodology, classifications of mole, case detection and definition of the denominator; the comparison of incidence of GTD among countries has been difficult. ${ }^{7}$ The recent attempts at standardization of histopathological and clinical nomenclature has improved uniformity in the different epidemiological studies across the world. ${ }^{7,8}$ Unlike few of the western countries, as India does not have the disease specific registry for gestational trophoblastic disease, only institution based studies are supposed to provide some light on the epidemiological data. Hence this study is an effort to understand the incidence, demographics, treatment and outcome of hydatidiform mole patients in a tertiary care government hospital.

The incidence of GTD in this study is observed to be 1.13 per 1000 deliveries. This is slightly lower than the rates observed in previous hospital based studies in Dubai (2.5 per 1000 live births) and Malaysia (2.6 per 1000 deliveries), but is comparable to the only institute based similar study from India (1.31 per 1000).$^{5,9,10}$ In a study conducted across races in Northern England and Wales, the incidence of gestational trophoblastic disease in the Asian population was found to be 1.95 times higher than in the non-Asian population (1 per 387 live births versus 1 per 752 live births). ${ }^{11}$ Majority of the patients belonged to age group 20-24 years (59.2\%), similar to the study by Kumar $\mathrm{N}$ et al; with the mean ages being similar as well. ${ }^{10}$ FIGO and WHO criteria states age more than 39 years as a high-risk factor; but this was not reflected in the present study as there were no patients with age more than even 35 years. This could be due to the small sample size, early age at marriage in the Indian society and attainment of maximum fertility at lower age itself. More than half of the patients were found to be below the age of 25 years. Age less than 20 years is not considered as a risk factor by WHO, though some experts have observed both extremes ( $<20$ years and $>35$ years) to be at increased risk of disease. ${ }^{12-15}$

In the present study the commonest symptom after amenorrhea is per vaginum bleeding, seen in $68.2 \%$ of patients. The clinical study from Dubai reported incidence of vaginal bleeding in $29 \%$ patients only. ${ }^{5}$ However, Fatima et al, noted bleeding per vaginum to be the commonest symptom seen in as many as $94.2 \% .^{14}$ One of the most classical symptom i.e. passage of grape like vesicles per vaginum was seen in only $9.1 \%$ of patients in the present study; while in the Nigerian study by Ocheke AN et al, it was found in $60 \%$ cases. ${ }^{13}$

The antecedent pregnancy was an abortion in $23 \%$ of patients. These numbers were comparable with many other studies. The study from Pakistan observed $36.5 \%$ of patients to be primigravidas. ${ }^{14}$ The other study from northern part of India had $21 \%$ primigravida among all patients and $26 \%$ had a history of abortion in antecedent pregnancy. ${ }^{10}$ However the Nigerian study had $48 \%$ of the patients with previous history of at least one abortion. ${ }^{13}$

The mean gestational age during diagnosis in the present study was $12.8 \pm 3.96$ weeks. This is comparable with many other recent studies, like Nirmala et al. having observed a mean gestational age of $11 \pm 3$ weeks during presentation. ${ }^{9}$ However Ocheke AN et al, reported a mean gestational age of 17 weeks during diagnosis. ${ }^{13}$ This is probably because the routine use of ultrasonography for the evaluation of early pregnancy has led to earlier diagnosis and the mean gestational age at presentation has decreased.

In the present study a total of $11(50 \%)$ women had a uterus larger than dates, which was similar to previous similar studies. $5,10,13,14$ The usually high prevalence of anaemia and subsequent requirement of blood transfusion in GTD cases was also observed, with $77.3 \%$ patients having $\mathrm{Hb}$ of $<10 \mathrm{gm} \%$ at the time of presentation and $81.8 \%$ participants requiring blood transfusion.

In the present study, the pre-evacuation $\beta$-hCG level was higher in complete mole group $(1.92 \pm 1.79$ lakhs IU/L) compared to partial mole group $(1.84 \pm 0.76$ lakhs IU/L), however the difference did not meet statistical significance $(\mathrm{p}=0.921)$, probably because of small sample size. Some similar studies with larger samples did note mean pre-evacuation serum $\beta$-hCG level to be significantly higher in the complete mole. ${ }^{16,17}$

The difference for number of weeks until the serum $\beta$ hCG level became undetectable was statistically not significant between complete and partial mole in this study $(\mathrm{p}=0.141)$, numerically higher in complete mole group though. Similar observations were made by Nirmala et al. ${ }^{9}$ 
In the present study histopathology examination (HPE) of $22.72 \%$ of the patients showed partial mole, and in $72.72 \%$ patients HPE showed complete mole. These proportions were not consistent with the published literature. The Malaysian study by Nirmala et al observed $46.1 \%$ patients to have CHM and $53.9 \%$ having PHM. ${ }^{9}$ Lybol et al, observed $30.2 \%$ having CHM, $44.5 \%$ of patients having PHM and in rest $11.6 \%$ the HPE was unspecified. ${ }^{18}$

Complications usually associated with GTN include haemorrhage, fever, septicemia, tumour embolization and uterine perforation. These were minimal in our study. One patient $(4.5 \%)$ had haemorrhage. One underwent hysterectomy in view of uterine perforation. Only one (4.5\%) patient developed GTN, that being invasive mole. Similar proportions were reported in the Malaysian study, where $3.9 \%$ patients developed persistent trophoblastic disease. ${ }^{9}$ However in the study by Kumar et al, $23 \%$ of the patients developed invasive mole and $14 \%$ developed choriocarcinoma. ${ }^{10}$

\section{CONCLUSION}

In conclusion, it can be said that early diagnosis and treatment is the key in GTD. Regular follow up of the patients is critically important for ensuring complete remission. Further, a multi-centric study is essential in India to determine the true incidence and overall outcome of molar pregnancy, which will help in the understanding exact burden of the disease. Because of paucity of population-based studies in India, there is imminent need to establish a centralised disease specific registry to ensure availability of unbiased and representative data.

Funding: No funding sources

Conflict of interest: None declared

Ethical approval: The study was approved by the Institutional Ethics Committee

\section{REFERENCES}

1. Seckl MJ, Sebire NJ, Berkowitz RS. Gestational trophoblastic disease. The Lancet. 2010;376(9742):717-29.

2. Matsui H, Iitsuka Y, Yamazawa K, Tanaka N, Seki $\mathrm{K}$, Sekiya S. Changes in the incidence of molar pregnancies. A population-based study in Chiba Prefecture and Japan between 1974 and 2000. Human Reprod. 2003;18(1):172-5.

3. Steigrad SJ. Epidemiology of gestational trophoblastic diseases. Best practice and research. Clin Obstet Gynaecol. 2003;17(6):837-47.

4. Smith HO, Qualls CR, Prairie BA, Padilla LA, Rayburn WF, Key CR. Trends in gestational choriocarcinoma: a 27-year perspective. Obstet Gynecol. 2003;102(5):978-87.
5. Rangwala TH, Badawi F. A profile of cases of gestational trophoblastic neoplasia at a large tertiary centre in Dubai. Obstet Gynecol. 2011;2011.

6. Kohorn EI. The new FIGO 2000 staging and risk factor scoring system for gestational trophoblastic disease: description and critical assessment. Int $\mathbf{J}$ Gynecol Cancer. 2001;11(1):73-7.

7. Palmer JR. Advances in the epidemiology of gestational trophoblastic disease. J Reprod Med. 1994;39(3):155-62.

8. Ngan HY, Odicino F, Maisonneuve P, Creasman WT, Beller U, Quinn MA, et al. Gestational trophoblastic neoplasia. Int $\mathrm{J}$ Gynecol Obstet. 2006;95:S193-203.

9. Nirmala CK, Nor Azlin MI, Harry SR, Lim PS, Shafiee MN, Nur Azurah AG, et al. Outcome of molar pregnancies in Malaysia: a tertiary centre experience. J Obstet Gynaecol. 2013;33(2):191-3.

10. Kumar N, Saxena YK, Rathi AK, Chitra R, Kumar P. Host and risk factors for gestational trophoblastic disease: a hospital-based analysis from India. Med Sci Monitor. 2003;9(10):442-7.

11. Tham BW, Everard JE, Tidy JA, Drew D, Hancock BW. Gestational trophoblastic disease in the Asian population of Northern England and North Wales. Int J Obstet Gynaecol. 2003;110(6):555-9.

12. Brinton LA, Bracken MB, Connelly RR. Choriocarcinoma incidence in the United States. Am J Epidemiol. 1986;123(6):1094-100.

13. Ocheke AN, Musa J, Uamai AO. Hydatidiform mole in Jos, Nigeria. J Nigeria Med Association. 2011;52(4):223.

14. Fatima M, Kasi PM, Baloch SN, Kassi M, Marri SM, Kassi M. Incidence, management, and outcome of molar pregnancies at a tertiary care hospital in Quetta, Pakistan. ISRN Obstet Gynecol. 2011;2011:925316.

15. Koirala A, Khatiwada P, Giri A, Kandel P, Regmi M, Upreti D. The demographics of molar pregnancies in BPKIHS. Kathmandu Univer Med J. 2011;9(4):298-300.

16. Feltmate CM, Batorfi J, Fulop V, Goldstein DP, Doszpod J, Berkowitz RS. Human chorionic gonadotropin follow-up in patients with molar pregnancy: a time for reevaluation. Obstet Gynecol. 2003;101(4):732-6.

17. Massad LS, Abu-Rustum NR, Lee SS, Renta V. Poor compliance with postmolar surveillance and treatment protocols by indigent women. Obstet Gynecol. 2000;96(6):940-4.

18. Lybol C, Thomas CM, Bulten J, van Dijck JA, Sweep FC, Massuger LF. Increase in the incidence of gestational trophoblastic disease in The Netherlands. Gynecol Oncol. 2011;121(2):334-8.

Cite this article as: Mulik J, Choudhary A. Clinical study of gestational trophoblastic disease in a tertiary care hospital. Int J Reprod Contracept Obstet Gynecol 2020;9:2964-8. 